\title{
Correction to: Vapor Nucleation in Metastable Liquids The Continuum Description
}

\author{
Mirko Gallo, Francesco Magaletti, Dario Abbondanza, \\ and Carlo Massimo Casciola
}

\section{Correction to:}

Chapter 12 in: M. Marengo and J. De Coninck (eds.), The Surface Wettability Effect on Phase Change, https://doi.org/10.1007/978-3-030-82992-6_12

The original version of this book was inadvertently published without including a chapter 12. The chapter 12 titled "Vapor Nucleation in Metastable Liquids The Continuum Description" has been added at the end of the book. 